\title{
MEDIOS TELEMÁTICOS PARA LA ENTREVISTA PERSONAL DE LAS PERSONAS CON DISCAPACIDAD INTELECTUAL EN EL PROCESO DE RESTRICCIÓN DE LA CAPACIDAD DE EJERCICIO
}

\section{TELEMATIC MEANS FOR THE PERSONAL INTERVIEW OF PEOPLE WITH INTELLECTUAL DISABILITIES IN THE PROCESS OF RESTRICTING THEIR EXERCISE CAPACITY}

\section{Juan Antonio Seda ${ }^{1}$}

DOI: https://doi.org/10.37767/2591-3476(2021)16

\section{Comentario a}

"S. J. V. S/ DETERMINACION DE LA CAPACIDAD JURIDICA" Juzgado de Familia $N^{\circ} 1$ de Tigre - Poder Judicial de la Provincia de Buenos Aires

Disponible en

https://bit.ly/38hY13J

RESUMEN:

El proceso de restricción de la capacidad de ejercicio para las personas con discapacidad mental o intelectual, requiere de una entrevista personal entre el causante y el magistrado a cargo de la causa. Esta entrevista no debería considerarse como una medida de prueba, ya que los jueces no tienen competencias profesionales para evaluar la salud mental o las limitaciones cognitivas de un individuo. En cambio, sí se la puede considerar como una forma de entablar un vínculo inmediato entre quien acude al sistema judicial y quien lo administra. En este comentario se analizarán algunos aspectos relacionados a la situación sanitaria vigente

\footnotetext{
1 Abogado (UBA); Doctor en Derecho (UBA). Profesor Adjunto Regular de Derecho de Familia y Sucesiones (UBA). Profesor Adjunto Regular de Práctica Profesional (UBA). Licenciado en Ciencias Antropológicas (UBA). Director de la Carrera de Especialización en Discapacidad y Derechos (UBA). Mail: juan. antonio.seda@gmail.com. ORCID iD: https://orcid.org/0000-0002-7362-2607
} 
en 2020 y 2021 y cómo esa inmediación, a su vez queda mediada por medios telemáticos. Esta alternativa puede constituir una vía para mantener contactos periódicos entre personas con discapacidad mental o intelectual y los equipos técnicos de los juzgados.

\section{ABSTRACT}

The process of restricting exercise capacity for people with mental or intellectual disabilities requires a personal interview between the deceased and the magistrate in charge of the case. This interview should not be considered as a test measure, as judges do not have the professional competence to assess an individual's mental health or cognitive limitations. On the other hand, it can be considered as a way of establishing an immediate link between who goes to the judicial system and who administers it. This comment will analyze some aspects related to the current health situation in 2020 and 2021 and how that immediacy, in turn, is mediated by telematic means. This alternative can be a way to maintain regular contacts between people with mental or intellectual disabilities and the technical teams of the courts.

PALABRAS CLAVE: Capacidad de ejercicio; restricción; proceso; inmediación; apoyos.

KEY WORDS: PExercise capacity; restriction; process; immediacy; supports.

\section{Introducción}

El proceso de restricción de la capacidad de ejercicio para las personas con discapacidad mental o intelectual tiene un primer objetivo, directo e inmediato, que es el de restringir la posibilidad de celebración de actos jurídicos por parte del causante, que pudieran luego ser tachados de nulos (por carecer de discernimiento quien los celebró). Se trata de una limitación que no afecta la personalidad jurídica del individuo, sino la facultad de ejercer por sí mismo esa capacidad. El segundo objetivo, complementario, es el de buscar un acompañamiento para que esa persona pueda celebrar aquellos actos jurídicos, pero con los apoyos necesarios y las salvaguardias que correspondan. El acceso a la justicia y la tutela judicial efectiva a personas que, por causa de una discapacidad mental o intelectual, están en situación de vulnerabilidad, requieren de un celoso apego a ciertas garantías procesales. Son causas en las cuales se necesita un especial cuidado en asegurar que sea escuchada la voz del titular del proceso, de allí que la legislación previó una instancia de inmediación en el trato entre magistrado y causante.

Tan importante es el respeto por la subjetividad de la persona con discapacidad mental o intelectual, que el legislador ha considerado especialmente la efectivización de una forma de comunicación, personal e intransferible. Así, una de las reformas incorporadas en el Código Civil y Comercial en este proceso es la obligatoriedad de una entrevista personal entre el causante y el magistrado que interviene en la determinación de la capacidad de ejercicio (y su eventual restricción). No se puede considerar que esa reunión reviste un carácter menor, por la trascendencia que la ley de fondo le otorga a su omisión. Tal es la magnitud de la importancia de este encuentro personal, que su falta de realización conlleva la nulidad del procedimiento. Inclusive se prevé que, ante la imposibilidad de asistencia del causante a la sede del juzgado, será el magistrado quien deberá trasladarse al lugar donde se encuentre la persona con discapacidad mental o intelectual, para llevar adelante la entrevista. 
En este comentario se analizará la modificación obligada que sufrió el formato de esta entrevista personal, a partir de la situación sanitaria vigente en 2020 y 2021. Las circunstancias sanitarias hicieron que esa reunión, en algunos casos, haya quedado mediada por medios telemáticos. Justamente, el primer punto a analizar en este sentido, es si el uso de esas tecnologías mantiene la noción básica de inmediación. Antes de avanzar sobre los debates que abre la mediación tecnológica en esa entrevista, deberíamos interrogarnos brevemente acerca de la naturaleza jurídica de ese encuentro personal. ¿Es una medida para mejor proveer? ¿Una oportunidad para la defensa de los derechos del causante? ¿Una notificación? ¿Una mera ceremonia protocolar? Algunos magistrados han planteado inclusive que la reunión es, en sí misma, la representación del llamado "acceso a la justicia", un término frecuentemente utilizado en documentos internacionales de derechos humanos, como las denominadas "Cien Reglas de Brasilia para el Acceso a la Justicia de las Personas en Condición de Vulnerabilidad". Si bien este documento lleva el título de "reglas", hay que aclarar que no son normas formales, sino solamente recomendaciones formuladas por un conjunto de jueces de diversos países, reunidos en el año 2008 en la décimo cuarta Cumbre Judicial Iberoamericana, en la ciudad de Brasilia. Se trata de un texto muy citado, pero que no constituye una norma de cumplimiento obligatorio. Sin perjuicio de ello, se puede tomar como derecho blando (soft law) y, por lo tanto, sin ser vinculante, no carece totalmente de significado legal.

Estas Reglas de Brasilia aspiran a aportar una interesante perspectiva de empatía y protección hacia los grupos más débiles de la sociedad, pero también hay que prevenir por algunos efectos perjudiciales para la calidad institucional, como es una interferencia de los jueces en la esfera del legislador, por medio del activismo judicial (Ramos, 2014; Calvinho, 2015). Nociones como el acceso a la justicia o la tutela efectiva constituyen principios, objetivos a lograr, ideas fuerza que marcan un clima de época y que se reiteran en diversos pronunciamientos de tribunales de diverso orden. Por ello, también las leyes sustantivas y adjetivas han tomado el compromiso de acercar a los funcionarios judiciales al público, de manera de obtener un servicio de justicia de mayor calidad y legitimidad.

Esta entrevista personal tiene varias dimensiones y quizás sería un error tratar de encasiIlarla en una única función dentro de un proceso tan peculiar. Hay aspectos de la noción de la inmediación que se explican mejor con el análisis situado en la práctica cotidiana, entendiendo el estilo que elige cada magistrado. Son muchos los jueces que asumen una comunicación directa con quien es circunstancial usuario del servicio de justicia y que, con sentido de responsabilidad por su tarea, se preocupan por entablar vínculos de empatía, siempre dentro de lo que su rol les permite. Hay un conocimiento que surge en estos encuentros, que se complementa de manera aleatoria con la información proveniente del expediente judicial y esto puede tener importancia para quien administra justicia, ya que no son pocos los magistrados que desarrollan una intuición que puede ser valiosa para el desarrollo de su función.

A primera vista, parece evidente que tal entrevista no podría considerarse como una medida de prueba, ya que los jueces no tienen conocimientos profesionales en ciencias médicas, neurológicas o de la conducta, como para evaluar la salud mental o las limitaciones cognitivas de un individuo. Claro que en algunos casos los magistrados pueden tomarlo como una especie de constatación personal de lo que han recibido en el informe del equipo interdisciplinario. Desde esa perspectiva, sí podría considerarse de manera amplia como una medida para mejor proveer, que pone al magistrado en contacto per- 
sonal con el causante y sus familiares. O sea que impacta en su convicción, pero también en el titular de este proceso voluntario y de sus familiares. A continuación, analizaremos brevemente un caso en el cual se recurrió a la realización de la entrevista personal por vía telemática.

\section{Desarrollo del comentario}

Hemos planteado hasta aquí, que todo el proceso de restricción de la capacidad de ejercicio debe tener un sustrato de protección y resguardo de garantías para las personas con discapacidad mental o intelectual, ya que se hallan en una condición de mayor dificultad o vulnerabilidad por su falta de discernimiento. Se ha dicho en muchos pronunciamientos judiciales que hay grupos de la población que requieren de una protección especial, tanto por parte de la ley como de sus familias (Medina, 2016). La carencia de discernimiento del causante en estos procesos, hace más relevante aún el cuidado en la tramitación y en cada detalle del procedimiento.

Aquí nos detendremos especialmente en una novedad específica que surge como producto del contexto del aislamiento social preventivo y obligatorio que derivó de la pandemia del COVID-19. El fallo analizado trata sobre un proceso de restricción de la capacidad de ejercicio de una persona adulta. Esta causa tramitó en el Juzgado de Familia $N^{\circ} 1$ de Tigre (Provincia de Buenos Aires) y la sentencia analizada es de julio del 2020, en medio de las restricciones establecidas por la situación sanitaria. El causante contaba con cuarenta y nueve años al inicio del proceso, que fue iniciado por su hermana, también mayor de edad. El patrocinio jurídico quedó a cargo de la Defensoría Oficial. Recordemos que la legitimación para instar estos procesos es limitada a los colaterales sólo hasta el segundo grado.

El diagnóstico de quien es titular del proceso es de retraso mental moderado, con un comportamiento que no puede considerarse adaptado a la vida social. La restricción de la capacidad de ejercicio para ciertos actos, tiene como propósito que el titular de los derechos no celebre determinados negocios jurídicos, que lo pudieren perjudicar o que simplemente podrían ser anulados, por carencia de discernimiento. En este caso, se dispuso una medida de inhibición general de bienes, de modo de evitar cualquier acto de disposición de su patrimonio. Se trata de un adulto que nació en una familia con una madre y cinco hermanos, aunque ha sido la hermana que inició el proceso quien desde muy pequeña se ocupó de los cuidados personales. En la actualidad, el causante, no tiene empleo y concurre a un Centro de Día y a un polideportivo municipal, como actividades de integración y de esparcimiento. Si bien su discapacidad intelectual es moderada, requiere de apoyos para las actividades de la vida diaria, de lo cual se ocupa su hermana.

Vemos aquí una situación bastante frecuente y es el inicio del proceso por parte del familiar que ya en los hechos llevaba adelante los apoyos ${ }^{2}$. Inclusive, de la lectura de la sentencia, surge que esta hermana es quien entiende lo que dice el causante y puede traducirlo. En las evaluaciones psicológicas se suele dejar constancia sobre detalles que marcan características de las limitaciones de la persona para interactuar socialmente, como por ejemplo la dificultad para entender el valor del dinero. También se evalúa si el individuo tiene habilidades para sostener una existencia autónoma, lo cual es fundamental para saber qué clase de apoyos se requerirán. Generalmente, esto suele plantearse

2 Los cuidados personales por parte de los familiares cercanos suelen ser la regla más habitual y los sistemas legales que controlan esas situaciones, pretenden evitar situaciones de abuso, tanto sea de un posible aprovechamiento de una influencia indebida, como también de las limitaciones fácticas que puedan tornarse en una sobre protección tiránica (Seda, 2021) 
nuevamente en la entrevista personal, lo cual le otorga un carácter híbrido porque si bien no es una medida de prueba, sirve a la magistrada para darse una idea por sí misma de las posibilidades fácticas del causante.

Un aspecto a destacar del fallo es que vincula la condición del causante con la categoría jurídica y política de "persona con discapacidad", que es mencionada atinadamente en los fundamentos de la sentencia. Hay que aclarar que el Código Civil y Comercial en sus artículos 48 y 2448 brinda una definición operativa de "persona con discapacidad". Esta definición propia del Código es de dudosa consistencia convencional, ya que existía otra en nuestro ordenamiento jurídico. Esta definición plantea que “...se considera persona con discapacidad a toda persona que padece una alteración funcional permanente 0 prolongada, física o mental, que en relación a su edad y medio social implica desventajas considerables para su integración familiar, social, educacional o laboral". Hay que decir que es similar a la que proporciona la Convención sobre los Derechos de las Personas con Discapacidad, pero no es la misma (tal Convención fue ratificada por nuestro país por medio de la Ley № 26.378 y obtuvo jerarquía constitucional, en los términos del inciso 22 del artículo 75 de la Constitución Nacional, por medio de la Ley N²7.044). Es necesario remarcar y enfatizar en que no tiene ninguna explicación razonable que el legislador argentino haya dado una nueva definición a la persona con discapacidad, cuando ya existe otra vigente, proveniente de un tratado internacional de derechos humanos ${ }^{3}$.

La idea de certificar la discapacidad por parte del Estado surgió en 1981, a través de la Ley № 22.431 y luego se actualizó en 2009, por medio de la Ley № 25.504. El Certificado Único de Discapacidad es un documento emitido por la Administración, sólo a pedido del interesado y que se gestiona en cada jurisdicción, aunque con parámetros comunes en todo el país. Estos estándares para medir la discapacidad, surgen de un documento emitido en el año 2001 por Organización Mundial de la Salud, denominado "Clasificación Internacional sobre el Funcionamiento de la Salud y de la Discapacidad". En este documento se evalúa la discapacidad, a partir de lo que se denomina un diagnóstico funcional y que contempla varias categorías: funciones corporales, estructuras corporales, actividad, participación y factores ambientales. En la Convención, los cuatro tipos genéricos de deficiencias mencionadas son: físicas, sensoriales, mentales e intelectuales.

La prueba de la condición de persona con discapacidad, por lo tanto, podría surgir del Certificado Único de Discapacidad (CUD), en tanto documento público, que se emite luego de una evaluación interdisciplinaria. Sin embargo, ese certificado no podría nunca reemplazar integralmente a la evaluación del equipo interdisciplinario que surge del artículo 37 del Código Civil y Comercial. El CUD solamente puede constituir una prueba documental más, a la cual eventualmente los peritos de la causa pueden consultar. Esto queda bien explicado en el fallo que se analiza, donde se produce prueba testimonial y pericial para dar cuenta cabal de las limitaciones en la vida cotidiana del causante. En este caso, se convocó a declarar a vecinos de la familia, que testimoniaron acerca del vínculo de cuidados que percibían de parte de la hermana al causante.

Uno de los aspectos a destacar en este proceso es en la utilización de medios digitales para

\footnotetext{
3 Este tema ha sido planteado ya en otras oportunidades, en el marco de un Proyecto de Investigación de la Universidad de Buenos Aires (UBACYT) denominado "Discapacidad y Derechos Sucesorios" y en publicaciones previas (Seda, 2017). La definición de personas con discapacidad tiene una connotación identitaria y nuestro país, al ratificar la Convención sobre los Derechos de las Personas con Discapacidad, debería utilizar las nomenclaturas que trae ese tratado internacional de derechos humanos
} 
suplir la presencialidad, pero mantener la inmediación. ¿Cómo se puede hacer una entrevista personal entre la magistrada y el causante durante el aislamiento social preventivo obligatorio, ordenado como producto de la pandemia? En la primera oportunidad posible, se hizo uso de los medios técnicos disponibles y se habilitó la opción de una audiencia por medio telemáticos, tal como había autorizado la Suprema Corte de la Provincia de Buenos Aires, a través de la Resolución N 480/2020. Por supuesto que para que ello fuera posible, se pidió la conformidad de su letrado patrocinante (en este caso a través de la Defensoría Oficial) pero además del Ministerio Pupilar. La entrevista se realizó a través de la aplicación Zoom, con la participación del causante, su hermana, su letrado patrocinante (Defensor Oficial), la representante de la Asesoría de Incapaces, la magistrada y personal de su juzgado.

\section{Conclusiones}

La utilización de medios telemáticos para la realización de la entrevista personal constituye, en este caso, un acierto que permitió avanzar con el proceso sin omitir ningún requisito. Podemos notar, a partir de lo referido en la sentencia, que la experiencia fue satisfactoria para la magistrada como así también para el propio causante y sus familiares. Si bien la persona con discapacidad intelectual cuya capacidad de ejercicio estaba en debate, no podía expresarse por sí mismo, sí interactuaba con la mediación de su hermana. Vemos mucho en la práctica forense esa clase de traducciones, producto de años de convivencia y vínculo de apoyo en la vida cotidiana. Entonces, la aparición de los medios telemáticos no puede significar una frustración para estas audiencias, más bien son una modalidad diferente y a la que se puede acudir cuando se haga imposible el encuentro por otro medio.

Hay que agregar que también esta tecnología abre la puerta a un seguimiento que no termine con la eventual declaración de una restricción y la designación de apoyos. Gracias a esta clase de soluciones, que son producto de desafíos que plantea el contexto mundial, podemos pensar en adaptaciones de esta entrevista personal, siempre con un criterio de brindar mayores garantías a las personas con discapacidad mental o intelectual. Por supuesto que no es lo mismo el contacto a través de los dispositivos informáticos que el contacto personal directo, pero ello no quita que no podamos considerar a estas entrevistas como "personales".

¿Cuáles son, a fin de cuentas, los componentes de la entrevista personal? ¿Qué clase de interacción se produce allí? De la consulta con muchos colegas y también con magistrados, podemos decir que los roles son variables, según la condición del causante, el entorno familiar, los potenciales conflictos económicos y la clase de cuidados personales que se requieran. En cualquier caso, la fugacidad del contacto y la trascendencia de los efectos no son proporcionales, sea a través de medios telemáticos o no. Esta forma de entablar un vínculo inmediato entre quien acude al sistema judicial y quien lo administra, condensa un contenido simbólico que no desaparece por el uso de la tecnología. Hay un valor intangible en ese encuentro, difícil de definir pero que cada juez y cada familia involucrada percibe. La ofrenda del tiempo de los magistrados, uno de los núcleos en que se apoya esa búsqueda de legitimidad del proceso, no necesariamente se diluye por la mediación tecnológica.

El propósito de brindar mayor participación al causante, así como informarlo de manera directa, se puede perfectamente llevar adelante a través de otros medios ${ }^{4}$. Las entrevis-

4 En estos años se han llevado a cabo experiencias valiosas en todo el país en este sentido, sólo por citar una de la ciudad de Concepción del Uruguay (Entre Ríos), mencionaré al juez Dante Abel Command, que ha desarrollado una serie de criterios para la preparación de las entrevistas y la lectura y explicación de la sentencia. 
tas por vía telemática pueden constituir una alternativa válida para mantener contactos periódicos entre personas con discapacidad mental o intelectual y los equipos técnicos de los juzgados. En cuanto a la evaluación técnica interdisciplinaria, fue llevada adelante por un equipo conformado por un médico psiquiatra, un psicólogo y un trabajador social, todos ellos dependientes del propio juzgado. De la sentencia no surge con tanto detalle cómo hicieron sus respectivas pericias, pero es posible que también se hiciera uso de los medios telemáticos para facilitar las comunicaciones.

Merece un párrafo aparte el cuidado del fallo en asegurar la "lectura fácil", una incorporación de dudosa eficacia, pero sobre todo de riesgosa inclusión en una sentencia. No son poco los casos de un agregado al fallo en el cual se trata de explicar lo mismo, pero en términos coloquiales, como si de esa forma el causante pudiera participar de un modo más activo en el proceso. Sin dudas que en la mayoría de los casos hay una noble intención, pero se corre el riesgo de caer en la incongruencia si hubiera cualquier clase de discordancia entre el texto original y fragmento presentado como de "lectura fácil".

Es posible que la entrevista personal sea una anomalía en términos procesales, una creación que todavía requiere del paso del tiempo para ver cómo los magistrados la aplican. Hemos visto que puede resultar de valor humano y social, más allá de no tener utilidad probatoria. La incorporación de la entrevista personal es uno de los cambios en este procedimiento, posiblemente valioso, pero no creo que tenga la suficiente entidad como para afirmar que estamos ante un "cambio de sistema" ni mucho menos de "modelo" o "paradigma". En general, la hipérbole retórica no es útil para el discurso jurídico preciso y consistente. En lugar de ello, esa clase de exageraciones valorativas desproporcionadas, incitan al ingenuo optimismo de creer que estamos ante un cambio revolucionario. Hemos visto que, en cambio, a veces las mejores respuestas surgen de la aplicación del sentido común y de la prudencia aplicada al caso concreto.

\section{REFERENCIAS BIBLIOGRAFICAS:}

- CALVINHO, Gustavo (2015) “Los derechos humanos en la teoría del proceso" 2013, disponible on line: http://gustavocalvinho.blogspot.com.ar/2013/03/los-derechos-humanos-en-la-teoria-del.html Consulta: 30/3/2015

- MEDINA, Graciela (2016) "La adaptación de la sociedad al envejecimiento", Revista Derecho Privado y Comunitario, Rubinzal Culzoni, Santa Fe.

- PÉREZ, Miguel, ELISEU CASTELL, Frigus y BELMONTE CALDERÓN, Laura (2004) "Deterioro cognitivo leve. Pruebas de cribado en Atención Primaria". Comunicación oral. 24 congreso de la SEMFYC. Sevilla, 2004.

- RAMOS, Glauco (2014) “Activismo vs. garantismo en el proceso civil: presentación del debate" Revista Latinoamericana de Derecho Procesal, N² 2, 2014

- SEDA, Juan (2017) Discapacidad y Derechos. Impacto de la Convención sobre los Derechos de las Personas con Discapacidad, Jusbaires, Buenos Aires.

- SEDA, Juan (2021) “Familias de personas con discapacidad intelectual. ¿Apoyos o sustitución?", Revista Iberoamericana de Bioética / n 15 / 01-10 [2021] Madrid. 